\title{
Evidências de métodos participativos para o planejamento urbano da cidade saudável
}

DOI: 10.20396/labore.v13i0.8657594

Submetido 21 nov. 2019.

Aceito 01 der. 2019.

Publicado 26 dez. 2019.

\author{
Ana Maria Girotti Sperandio \\ $<$ https://orcid.org/0000-0002-9373-7727> \\ Universidade Estadual de Campinas / Campinas [SP] Brasil \\ Rodrigo Brandini Bloes \\ $<$ https://orcid.org/0000-0003-1410-652X> \\ Universidade Estadual de Campinas / Campinas [SP] Brasil \\ Pamela Shue Lang Lin \\ $<$ https://orcid.org/0000-0002-2575-0129>
}

RESUMO

Estudar e pesquisar princípios, valores e características de uma cidade saudável é um processo que demanda imersão. É necessário um entendimento do que é saudável ou ainda percorrer um caminho investigativo do que poderia ser o oposto disso, a fim de esclarecer a definição e aproximar da complexidade do conceito de saúde. Notando que o ambiente urbano é de considerável multiplicidade, as diversas instâncias que compõem a cidade saudável podem ser analisadas simultaneamente. Este artigo tem como objetivo apontar métodos participativos e as suas respectivas evidências para subsidiar as tomadas de decisões dos gestores públicos, no seu cotidiano, para o desenvolvimento da cidade saudável. A metodologia para tal apontamento baseou-se em revisões bibliográficas em relação aos temas, em leitura com profundidade dos documentos gerados nas cidades, após desenvolvimento dos projetos com atuação da academia, gestão pública e comunidade, diálogos das visitas técnicas realizadas nas cidades e registros fotográficos. Como resultados, o LABINUR/FEC/UNICAMP, a partir do monitoramento das experiências, tem obtido elementos para a formulação de uma metodologia do Planejamento Urbano Baseado em Evidências para a Cidade Saudável que poderá ser indicada para os gestores públicos com o intuito de relacionar a escolha ideal das iniciativas a serem postas em prática e trazer a participação social para o processo de tomada de decisões. Concluiu-se que o monitoramento dos métodos participativos permite aproximar da realidade social vivenciada em micro espaços e identificar evidências que podem ser significativas no processo de construção de políticas públicas para a cidade saudável.

\section{PALAVRAS-CHAVE}

Planejamento Urbano. Cidade Saudável. Promoção da Saúde. Evidências.

\section{Evidences of participatory methods for healthy city urban planning}

\begin{abstract}
Studying and researching the principles, values and characteristics of a healthy city is a process that demands immersion. It is necessary an understanding of what is healthy or to follow an investigative path of what could be the opposite of it, in order to clarify the definition and to approach the complexity of the concept of health. Noting that the urban environment is of considerable multiplicity, the various instances that make up the healthy city can be analyzed simultaneously. This article aims to point out participatory methods and their respective evidence to support the decision-making of public managers, in their daily accomplishments, for the development of a healthy city. The methodology for such an appointment was based on bibliographic reviews in relation to the themes, in-depth reading of the documents generated in the cities, after the development of projects with academy, public management and community performance, dialogues of the technical visits made in the cities and photographic records. As a result, LABINUR / FEC / UNICAMP, through the monitoring of experiences, has obtained elements for the formulation of an Evidence-Based Urban Planning methodology for the Healthy City that can be indicated to public managers in order to relate the ideal choice of initiatives to be implemented and bring social participation to the decision-making process. It was concluded that monitoring participatory methods allows the approach to the social reality experienced in micro spaces and identify evidence that may be significant in the process of building public policies for the healthy city.
\end{abstract}

\section{KEYWORDS}

Urban planning. Healthy City. Health promotion. Evidence. 


\section{Introducãa}

É primordial para o desenvolvimento de uma cidade saudável a aplicabilidade de métodos participativos que podem ser utilizados por gestores, comunidade e a academia a fim de conduzir o planejamento urbano nas suas diferentes fases, da concepção à implementação, consubstanciados pelo monitoramento e revisão constante.

Ações, projetos e iniciativas exitosas e transformadoras na cidade, contempladas pela participação da comunidade e de ações intra e intersetoriais que almejam a autonomia coletiva, dentre outros fatores, serão abordados neste artigo. Estes elementos podem indicar evidências positivas para uma gestão da cidade saudável.

A incorporação de ferramentas participativas na formulação da tomada de decisões é fator determinante para tornar a gestão da cidade eficiente. Os autores Snowden e Boone (2007) expõem a necessidade de um gestor, independentemente do setor em que atue, considerar um método para fundamentar sua tomada de decisão. Os autores apresentam uma estrutura por eles desenvolvida, nomeada como Cynefin, a qual permite apreender cinco graus de contextos para a tomada de decisão: simples, complicado, complexo, caótico e desordem (Snowden \& Boone, 2007). A fim de ilustrar os diferentes graus citados, Snowden e Boone (2007) mencionam diversas situações provindas do setor público e da iniciativa privada. Estes autores elencam, também, características que definem o sistema complexo, tais como: interações entre elementos; viabilizar sistema dinâmico e sinérgico, cuja solução não pode ser imposta e contém soluções emergenciais; o passado é integrado com o presente e os elementos se relacionam entre si e entre o ambiente; e o agente e o sistema restringem um ao outro, especialmente ao longo do tempo (Snowden \& Boone, 2007). Esses fatores possibilitam inferir sobre a sua correspondência com a dinâmica da cidade contemporânea.

A estrutura Cynefin aponta os métodos a serem adotados na formulação das resoluções para contextualizações complexas, que incluem dentre outras, a "abertura para discussão", a qual define a necessidade de realizar discussões democráticas, multidirecionais e interativas; e "encorajar a dissen-são e diversidade", com o intuito de formar padrões através das discussões entre distintos pontos de vista (Snowden \& Boone, 2007).

Ambos os métodos possuem caráter participativo, trazendo para o processo da formulação da tomada de decisão elementos que incluem a premissa de que para atingir a compreensão de algo complexo, há a necessidade de considerar as múltiplas concepções ao seu redor para que haja a troca de entendimentos, com a intenção de formar a compreensão do todo (Streck, 2016).

Em detrimento das diversas esferas de atuação do gestor público para realizar o desenvolvimento urbano através da gestão que atue democraticamente considerando a participação da sociedade (Lei n. 10.257, 2001), requisito básico instituído no Estatuto da Cidade, existe a necessidade de admitir a complexidade da realidade urbana, a qual possui uma rede de sistemas dinâmicos dotados de "diferentes subsistemas econômicos, sociais e ambientais" (Weiss, 2017, p. 808).

O dinamismo é uma característica implícita do processo de tomada de decisão, cuja estrutura está mudando ao longo do tempo e em função de seu contexto, processo esse que define a governança (Barten et al., 2011). De acordo com Barten et al. (2011), a análise da governança deve enfocar nos agentes envolvidos em processos de tomada de decisão coletiva, assim como nas estruturas estabelecidas para assegurar a efetividade na implementação (Barten et al., 2011). Desta forma, a governança em uma cidade saudável é subsidiada pelos métodos participativos tendo em vista da análise dos agentes, dos processos e das estruturas, evidenciando o seu movimento.

Corroborando com esta perspectiva, a Faculdade de Engenharia Civil, Arquitetura e Urbanismo, da Universidade Estadual de Campinas (FEC/UNICAMP), por meio do Programa de Pós- Graduação em Arquitetura, Tecnologia e Cidade, onde está a linha de pesquisa "Cidade: Planejamento e Projeto Urbano", tem estudado e pesquisado os condicionantes, as estruturas e os processos do espaço urbano em diferentes escalas e abordagem multidisciplinar, com o objetivo de colaborar com a formulação propositiva de novas teorias, cenários e projetos. No contexto desta linha de pesquisa, foi inserida a temática: "Estudos e pesquisas de metodologias sobre planejamento urbano para cidades saudáveis", juntamente ao Laboratório de Investigações Urbanas (LABINUR) da FEC/UNICAMP, que vêm promovendo e fomentando pesquisas, reflexões, traçados e concepções da cidade saudável e do planejamento urbano, constatando a suas coadunações e interdependências.

Este artigo tem como objetivo apontar métodos participativos e as suas respectivas evidências para subsidiar as tomadas de decisões dos gestores públicos, no seu cotidiano, para o desenvolvimento da cidade saudável. 


\section{2. cidade Saudável e Planejamento urbano: convergências, consonâncias, princípios e fatos históricos}

Estudar e pesquisar princípios, valores e características de uma cidade saudável é um processo que demanda imersão. É necessário um entendimento do que é saudável ou ainda percorrer um caminho investigativo do que poderia ser o oposto disso, a fim de esclarecer a definição e aproximar da complexidade do conceito de saúde. Notando que o ambiente urbano é de considerável multiplicidade, as diversas instâncias que compõem a cidade saudável podem ser analisadas simultaneamente.

De acordo com Corburn (2017), o planejamento é uma disciplina que inclui processos políticos, instituições e discursos que geram políticas, regras e planos urbanos que modelam onde vivemos, aprendemos e temos lazer nas cidades. Dessa forma, este ajuda a estruturar a distribuição dos bens sociais, físicos e econômicos que influenciam a saúde humana e explicam iniquidades (Corburn, 2017). Em vista disso, métodos de planejamento que utilizem ferramentas promotoras da qualidade de vida podem contribuir para a aproximação do ambiente urbano e a abordagem do conceito de cidade saudável.

Um dos documentos que caracteriza, qualifica e subsidia a cidade saudável é a Carta de Ottawa de 1986 que pontua em seu texto condições e recursos fundamentais para a saúde, entendidos como pré-requisitos, como: saúde, abrigo, educação, alimentação, recursos econômicos, ecossistema estável, recursos sustentáveis, justiça social e equidade (Ministério da Saúde, 2002). Estes estão em consonância com as premissas relativas à ocupação adequada do meio urbano, as quais são apresentadas nos marcos legais, tal como o Estatuto das Cidades, lei federal Brasileira $n^{\circ}$ 10.257/2001, o qual estabelece em seu artigo primeiro que a regulação da propriedade urbana deve ser feita em prol ao bem coletivo, da segurança e bem-estar dos cidadãos; e em seu artigo segundo que, dentre as diretrizes gerais para a política urbana, institui a garantia e direito às cidades sustentáveis, à gestão democrática e à oferta de equipamentos urbanos e comunitários de interesse da população (Lei n. 10.257, 2001).

A revisão da Política Nacional de Promoção da Saúde (2014) no Brasil, apresenta um conjunto de estratégias e formas de se produzir individual e coletivamente a saúde, no qual seus preceitos são baseados em princípios e valores fundantes para o seu processo de implantação e implementação (Política Nacional de Promoção da Saúde, 2014). Este documento fortalece a relação entre saúde e ambiente ao estabelecer como diretriz, por exemplo, em seu artigo quinto, o reconhecimento de contextos locais e o respeito à diversidade, que favoreçam a promoção de ambientes saudáveis pela construção de espaços de produção social, e reiterado pelo artigo sétimo, como objetivo específico favorável ao bem-viver e ao desenvolvimento humano (Política Nacional de Promoção da Saúde, 2014). A tomada de decisão dos gestores deve ser pautada nos aspectos anteriormente pontuados que transversalizam ações de promoção da saúde para a conexão com o planejamento urbano saudável.

Segundo Sperandio, Malek-Zadeh, Arêas e Francisco Filho (2019), uma das qualidades da cidade saudável é aquela que o indivíduo se sente pertencendo e que incorpora as seguintes caraterísticas: participação social, senso de pertencimento, identificação de partes interessadas, autonomia, coalizão, governança e equidade (Sperandio et al., 2019). Este pensamento da autora foi baseado em De Leeuw, 2017. Estas particularidades são corroboradas pelo planejamento urbano, uma vez que devem ser ferramentas aplicadas às cidades e que podem contribuir com o desenvolvimento das suas positividades e potencialidades.

Desde o final do século XIX, estudiosos realizam esforços para observar, refletir e propor soluções para a ocupação e expansão territorial urbana. Observações com foco na saúde dos cidadãos, indicaram relações entre as características do meio e as condições citadinas. Mapas foram produzidos com a intenção de localizar a doença, promovendo um movimento na direção do planejamento com um viés sanitarista, o que resultou no desenvolvimento e execução de planos de reforma urbana como a Paris de Haussmann, na Europa, e o Rio de Janeiro do Plano Agache, no Brasil.

Os esforços realizados para a projeção ideal da ocupação humana foram diversos: das cidades jardins de Ebenezer Howard (Domhardt, 2012) às cidades compactas de Richard Rogers (Rogers \& Gumuchdjian, 2001) e dos conceitos de Habitar, Circular, Trabalhar e Recrear da Carta de Atenas (Domhardt, 2012) às mais recentes metas dos Objetivos do Desenvolvimento Sustentável <https://nacoesunidas.org/ pos2015/agenda2030/>. É observado nesta breve linha do tempo que existem esforços multidisciplinares e intersetoriais para o estabelecimento de diretrizes que convergem a promoção da saúde com o planejamento urbano, enfatizando a importância da participação social e a autonomia coletiva para a governança na cidade, consequentemente, resultando na elaboração de políticas públicas saudáveis. 


\title{
3. Métodos participativos como meio para subsidiar a gestão pública da cidade saudável: as evidêencias
}

Para o desenvolvimento deste artigo foi realizado uma relação entre o planejamento urbano para uma cidade saudável e a metodologia utilizada na Medicina Baseada em Evidências (MBE) para qualificar a tomada de decisão dos gestores e representantes nas cidades.

Segundo Atallah (2018), a MBE tem como determinante para escolha da resolução adequada para tomada de decisões no projeto da clínica médica, aquela que apresenta evidências oriundas de processos metodológicos confiáveis e discriminadas em função de sua efetividade, eficácia, eficiência e segurança (Atallah, 2018; El Dib, 2007). Lopes (2000) corrobora que a MBE apresenta similitudes com a metodologia de ensino e aprendizado baseado em problemas, o que permite o desenvolvimento do raciocínio científico, novas atitudes para aprendizado e a interdisciplinaridade de conhecimentos em diferentes áreas (Lopes, 2000). As habilidades e competências para a aplicação da MBE, segundo Lopes (2000), são:

\begin{abstract}
Identificar problemas relevantes do paciente; converter os problemas em questões que conduzam às respostas necessárias; pesquisar eficientemente as fontes de informação; avaliar a qualidade da informação e a força da evidência, favorecendo ou negando o valor de uma determinada conduta; chegar a uma conclusão correta quanto ao significado da informação; aplicar as conclusões dessa avaliação na melhoria dos cuidados prestados aos pacientes (Lopes, 2000, p. 285).
\end{abstract}

O processo de tomada de decisão, seja no planejamento urbano na cidade, ou no campo da medicina, requer um estudo para a escolha dos métodos que considerem as características das demandas apresentadas e dos agentes envolvidos.

Por meio de experiências monitoradas pelo LABINUR/FEC/UNICAMP, identificou-se algumas evidências que podem subsidiar, teoricamente, a tomada de decisões para o desenvolvimento do planejamento urbano da cidade saudável, no qual os pesquisadores do LABINUR, ainda que em processo de pesquisa, propõem a utilização dos conceitos, princípios e valores de Promoção da Saúde, da MBE (Atallah, 2018; Lopes, 2000) e de planejamento urbano (Lei n. 10.257, 2001), convergindo para uma proposta de Planejamento Urbano Baseado em Evidências para a Cidade Saudável (PUBECS).

Segundo Lopes (2000), a realização da MBE requer algumas competências, as quais apresentam semelhanças conceituais para o processo de desenvolvimento do PUBECS e que traduzem as particularidades possíveis que um gestor público pode perceber para a tomada de decisão na direção de uma cidade saudável. Características estas, como: capacidade de distinguir demandas sociais em um contexto; converter os problemas em questões que possibilitem identificar quais métodos podem ser aplicados (com a identificação fundamentada nas respectivas evidências); realizar a avaliação da qualidade das informações e finalmente, o monitoramento da implantação e implementação dos métodos e ferramentas, a fim de observar a efetividade durante o processo.

É necessário considerar os conhecimentos adquiridos coletivamente dentro das comunidades para compor o processo de capacitação da gestão pública na cidade, bem como, o desenvolvimento de práticas participativas que possibilitem as pessoas serem protagonistas na promoção da saúde. Desta forma, a academia, em consonância com a atuação de redes de municípios, pode desenvolver pesquisas a fim de facilitar a identificação das evidências representativas; relatar e apresentar as boas iniciativas participativas; descrever as atuações dos agentes envolvidos e transformar os dados em informações acessíveis aos gestores públicos, possibilitando a aproximação e o fortalecimento da tríade: gestão pública, academia e comunidade, contribuindo assim para o desenvolvimento do PUBECS.

\section{Relatos das Experiências de métodos participativos na perspectiva da cidade saudável}

Os métodos participativos permitem aproximar a realidade social vivenciada pelas pessoas de um determinado local na cidade, com o desenvolvimento de potencialidades dos participantes. Os espaços criados possibilitam a coesão entre a cidade e pessoas no sentido ampliado do bem viver.

As experiências registradas academicamente neste artigo relatam ações que se relacionam as características da cidade como relatam Freire, Salles e Franco de Sá (2016) por meio de pesquisas realizadas na região nordeste do Brasil identificaram como ações promotoras de municípios saudáveis aquelas que contemplam a participação social como: a produção de artesanato; o núcleo de apoio às mulheres na prevenção de violência de gênero; o ambiente saudável na comunidade/ escola e o alimentação solidária. Na região do sudeste brasileiro, os estudos realizados por Sperandio et al. $(2015,2018,2019)$ identificaram ações coletivas e partici- 
pativas como diferenciais para a nova concepção da cidade saudável e que corroboram com o planejamento urbano (Lei n. 10.257, 2001) e a MBE (Atallah, 2018), como: a horta comunitária, a academia da saúde, a reciclagem de resíduos, dentre outros.

As iniciativas acima citadas são possíveis de serem implantadas e implementadas através das características dos métodos participativos, pois compreendem desde o envolvimento da gestão local e existência de lideranças locais comprometidas, até a criação de tecnologia social construída coletivamente, como é definido o Método Bambu (Freire, Salles \& Franco de Sá, 2016); assim como a existência de um projeto intersetorial, com a participação na Rede de Municípios Potencialmente Saudáveis (RMPS) 1 e a atuação conjunta dos agentes da comunidade (Sperandio, Moreira \& Bernardino, 2018). Essas características atuam de forma intra $\mathrm{e}$ intersetorial para promover a perenidade dessas iniciativas.

A seguir, serão apresentados três exemplos de metodologias participativas, estudadas, pesquisadas e monitoradas pelo LABINUR/FEC/UNICAMP com o apoio da RMPS e dos gestores envolvidos, que mediante critérios discutidos coletivamente, foram identificadas evidências de participação social na concepção da cidade saudável.

O primeiro exemplo trata do pré-teste de ferramenta participativa, o Mapa dos Desejos Locais (Sperandio et al., 2019), que apontou a possibilidade da leitura comunitária, e ao ser adotada pode possibilitar ao gestor público se adequar às competências necessárias para a aplicação do PUBECS; nos exemplos segundo e terceiro, são apresentadas iniciativas participativas, onde a transformação do espaço urbano e participação dos diferentes agentes pode indicar evidências de promoção da cidade saudável.

Este estudo aqui apresentado foi baseado em revisões bibliográficas em relação aos temas; na leitura de documentos gerados nas cidades, pós desenvolvimento dos projetos com atuação da academia, gestão pública e comunidade; resultados de diálogos de visitas técnicas nas cidades e registros fotográficos. Para identificar projetos geradores destas evidências, foram propostos alguns princípios e valores, tais como: participação social; envolvimento dos gestores, comunidade e academia; cooperação; elaboração de novas políticas públicas a partir de resultados dos diferentes projetos; reverberações das iniciativas em micro e sua repercussão em macro escala; multiplicação do conhecimento intra e inter-regional; resgate das culturas locais; perenidade e promoção de felicidade e afetividade. As experiências citadas foram numeradas e estão a seguir:

\subsection{O Mapa dos Desejos Locais no município de Holambra [SP]}

A ferramenta Mapa dos Desejos Locais, atualmente em processo de estudos e pesquisas pelo LABINUR no Município de Holambra [SP], onde foi realizado o pré-teste, demonstrou capacidade de desenvolver a participação social, realizar a interação entre comunidade e governo local, gerar informações a serem consideradas na concepção do Plano Diretor para a promoção da cidade saudável e ser um instrumento para o monitoramento de políticas locais. Segundo Sperandio et al. (2019), este instrumento foi desenvolvido com a expectativa de favorecer a democracia participativa para o desenvolvimento de cidades saudáveis, e com isso permite uma atmosfera propícia para a exteriorização dos desejos dos diferentes agentes da sociedade local e evidencia valores comuns para corroborar com a formação de uma coalizão entre os setores envolvidos.

A apresentação mapeada das informações, apresenta de modo objetivo os desejos da comunidade local do centro e da área rural (Sperandio, 2019). Assim, foi possível obter elementos para identificar práticas para a concepção da tomada de decisão, contribuindo para os estudos das competências necessárias do gestor público para o desenvolvimento PUBECS no LABINUR.

\subsection{A Horta COMUnitária de CONCHAL [SP]}

Uma prática participativa que apresentou resultados multidimensionais e correlacionados com os conceitos da cidade saudável é a Horta Urbana do Bairro Jardim Planalto, município de Conchal [SP]. No ano de 2010, a prefeitura local doou um terreno de aproximadamente 9 mil $\mathrm{m}^{2}$, antes desocupado, propício à marginalização, visualmente degradado, e que servia constantemente como depósito de resíduos, para a implantação da horta comunitária como estratégia de ocupação de vazio urbano, possível através da experiência da RMPS, do engajamento da comunidade e poder público local (Sperandio, Francisco Filho, Favero, Ribeiro \& Manfrinato, 2015).

A cidade de Conchal possui aproximadamente 28 mil habitantes (Instituto Brasileiro de Geografia e Estatística, 2019) e integra desde o ano de 2010 a RMPS. Neste mesmo ano foi instituída legislação

\footnotetext{
${ }^{1}$ A RPMS desde 2003 tem como função primordial subsidiar e instrumentar gestores públicos na concepção e implantação das cidades saudáveis com o enfoque no planejamento urbano. <http://www.redemunicipiosps.org.br>
} 
pertinente a programas de políticas públicas saudáveis (Lei n. 1761, 2010) o que favoreceu a implementação da horta comunitária, corroborando com a importância de a gestão pública articular ações que estejam embasadas nas premissas da cidade saudável e utilizar da sua função social para construir políticas públicas na direção da promoção da saúde.

Ao decorrer dos anos a horta apresentou fortalecimento com a adesão do número de participantes. Utilizada pela comunidade local, teve sua implantação realizada por onze famílias, as quais foram contempladas através de sorteio. No ano de 2015, vinte e quatro famílias participantes do projeto produziam alimentos naturais orgânicos. Atualmente o projeto continua e demonstra perenidade, sustentabilidade e envolvimento da comunidade (Prefeitura Municipal de Conchal, 2018).

Com o monitoramento do LABINUR e através dos estudos realizados como resultados práticos das disciplinas de pós-graduação Planejamento Urbano como promotor da Saúde da Cidade Saudável, Tópicos Especiais oferecidos nos anos de 2014 a 2018 na FEC/UNICAMP, foram efetuadas avaliações de pós ocupação (APO), por exemplo, utilizando-se a ferramenta Walkthrough (Rheingantz et al., 2009), com o intuito de avaliar a reverberação da horta na vida da comunidade local em Conchal-SP e quais eram os desejos das comunidades e dos representantes da gestão pública para a cidade de Holambra [SP] (Sperandio et al., 2015; Sperandio et al., 2019).

Em ambas as aplicações foram obtidos resultados transversais aos valores de promoção da saúde, tais como solidariedade, felicidade, corresponsabilidade, humanização, inclusão social, e aos seus princípios, como participação social, empoderamento, intersetorialidade, integralidade e territorialidade (Política Nacional de Promoção da Saúde, 2014). Estes resultados puderam ser observados por meio das manifestações dos próprios moradores durante as atividades desenvolvidas nas disciplinas que remetem à horta como um equipamento público essencial para as suas vidas (Sperandio et al., 2015).

Com o processo de avaliação foi possível identificar as reverberações da implementação da horta comunitária, sua efetividade no caráter de realizar a função social da propriedade urbana, promover princípios e valores transversais na população local e transformar a realidade social positivamente em direção às premissas da cidade saudável. Estes resultados demonstram que esta iniciativa promove os nove critérios necessários para classificá-la como ferramenta para o desenvolvimento do PUBECS no LABINUR.

\subsection{O Horto de Plantas Medicinais, Aromáticas e Condimentares de Santa bárbara D’OESTE [SP]}

O Horto de Plantas Medicinais, Aromáticas e Condimentares desenvolvido no município de Santa Bárbara D'Oeste [SP], revelou ser uma iniciativa eficiente para a promoção de Cidades Saudáveis. O desenvolvimento de um espaço multidisciplinar, possibilitou a implantação de um horto, no ano de 2013, em uma Escola Municipal2 ${ }^{2}$ com a participação dos diversos setores, tais como Secretarias Municipais de Santa Bárbara D’oeste, Universidade de São Paulo através da Escola Superior de Agricultura Luiz de Queiroz (ESALQ/ USP) e a RMPS. Segundo Sperandio, Rosa e Carvalho (2017), esta iniciativa realizou movimentos relacionados com a promoção da saúde e a sua inter-relação com os campos da educação e da justiça social. Foram desenvolvidas experiências pedagógicas com a vivência no cultivo das plantas medicinais e as suas aplicações na vida diária destacando os benefícios que a mudança alimentar pode proporcionar à saúde, e viabilizando a participação social além do envolvimento com os alunos, os pais, professores e comunidade local (Sperandio, et al., 2017).

Desse modo, foi possível o aproveitamento de um vazio urbano para a promoção e proteção da saúde com enfoque na cidade saudável. O referido projeto, além de ser matéria do noticiário regional, também obteve reconhecimento internacional. No ano de 2017, ganhou o primeiro lugar no Prêmio Inovação, atribuído pela Antenna Technologies Foundation, uma fundação suíça que tem como objetivo descobrir iniciativas de promoção da saúde para países em desenvolvimento <https://www.antenna.ch/en/>.

Por meio da RMPS, os municípios participantes realizam o intercâmbio de experiências exitosas e facilitam a sua reverberação intra cidade e inter região. Os resultados ocorridos a partir da implantação do horto medicinal, mesmo que em uma escala micro, apresentam conexão com os Objetivos do Desenvolvimento Sustentável < https://nacoesunidas.org/pos2015/agenda2030/> e revelam o potencial, ao longo dos anos, de serem reproduzidas em uma escala macro e intrasetorial. A Figura 1 apresenta um infográfico apontando os ODS observados na implementação do projeto.

\footnotetext{
${ }^{2}$ Escola Municipal de Ensino Fundamental e Educação Infantil (EMEFEI) Prof. ${ }^{a}$ Maria Augusta Canto Camargo Bilia.
} 


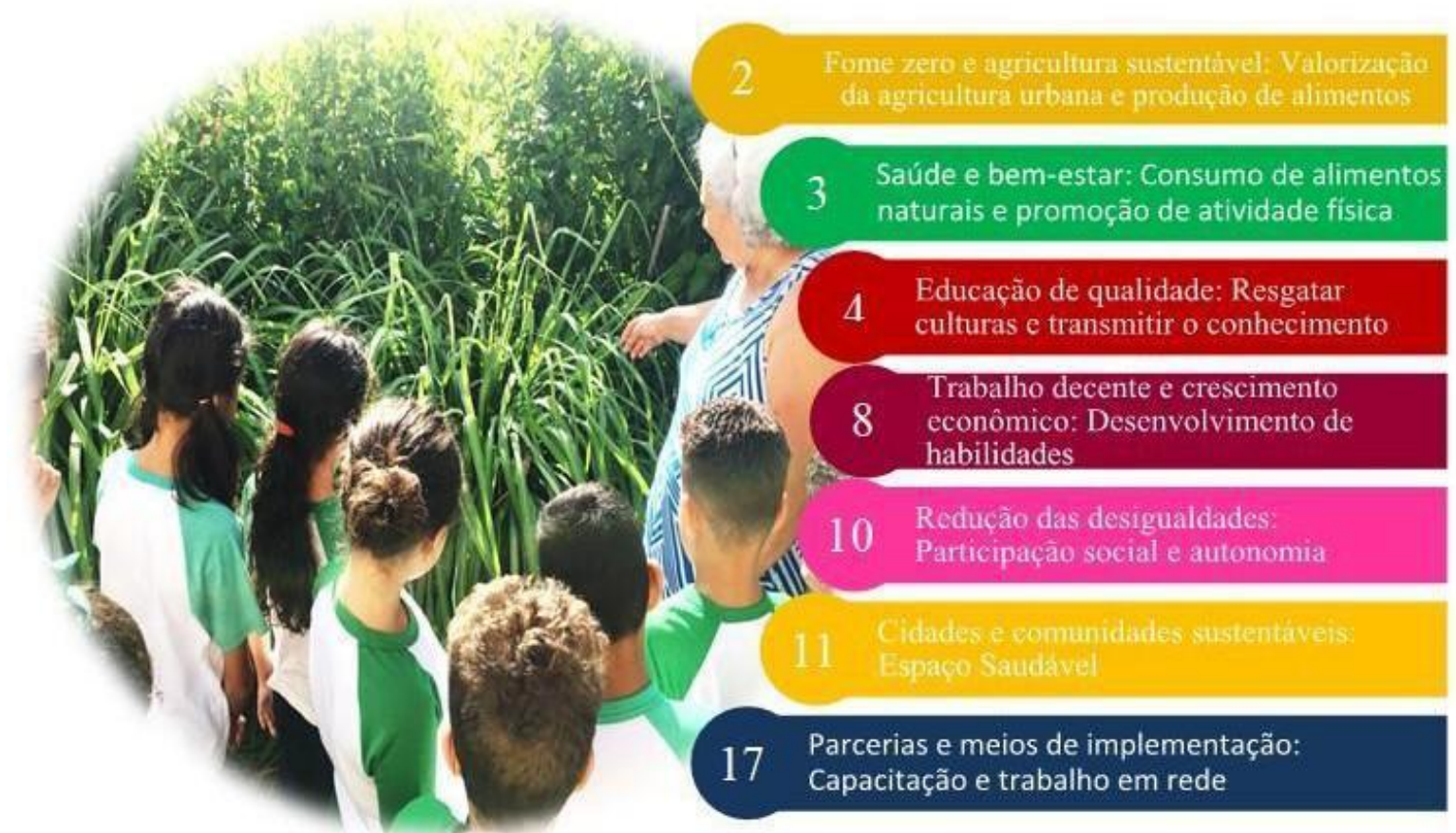

Figura 1. Infográfico que indica a aproximação entre Promoção da Saúde, Planejamento Urbano, MBE e os ODS - Horto de Plantas Medicinais, Aromáticas e Condimentares - Santa Bárbara D’Oeste, 2019. Fonte: LABINUR/FEC/UNICAMP.

A intenção dos pesquisadores na elaboração deste infográfico foi realizar, visualmente, a conexão da atividade em micro com a PNPS, o Planejamento Urbano, a MBE e os Objetivo do Desenvolvimento Sustentável (ODS). No que se refere às ações voltadas ao cumprimento das ODS, a atividade de cultivo atende ao Objetivo 2 - Fome Zero e Agricultura Sustentável, mediante a produção de alimentos saudáveis; contribui para o Objetivo 3 - Saúde e Bem-estar, não só pela alimentação e a atividade física, mas também ao acesso às terapias alternativas. A transmissão de conhecimento sobre as técnicas empregadas no cultivo, colabora com o Objetivo 4 - Educação de qualidade, pois resgata e transmite conhecimentos e cultura. O resultado do cultivo ao gerar renda, remete ao Objetivo 8 - Trabalho decente e Crescimento econômico, assim como o Objetivo 10 - Redução das desigualdades, por propiciar participação social e autonomia. A ocupação dos espaços para a realização da atividade, espaços esses que previamente eram vazios urbanos que não cumpriam sua função social, favorece o desenvolvimento do Objetivo 11 - Cidades e Comunidades Sustentáveis, e seu sucesso local replicado posteriormente por outros municípios contempla o Objetivo 17 - Parcerias e Meios de implementação, confirmando a efetividade do trabalho em rede. Estes fatos propiciam a conexão entre Promoção da Saúde, Medicina Baseada em Evidências e Planejamento Urbano, para além de contemplarem os ODS.

As evidências geradas pelo projeto apresentam sinais relacionados aos critérios estabelecidos para a identificação de uma ferramenta para a PUBECS, podendo classificar o horto como instrumento de promoção da cidade saudável.

As Figuras 2, 3 e 4 indicam, em síntese, os critérios estabelecidos pelos pesquisadores tendo em vista as experiências desenvolvidas nas cidades estudadas e monitoradas pelo LABINUR e apresentadas neste artigo (em cinza, critérios não atendidos).

Figura 2. Infográfico de identificação dos critérios na experiência de aplicação do Mapa dos Desejos Locais no município de Holambra [SP], em 2019.

Fonte: LABINUR/FEC/UNICAMP.

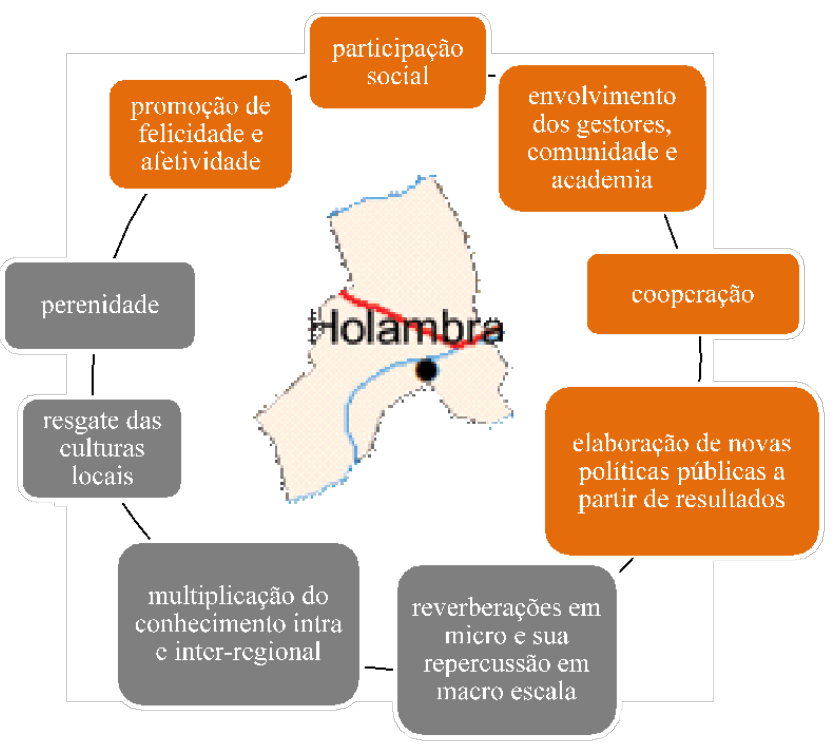



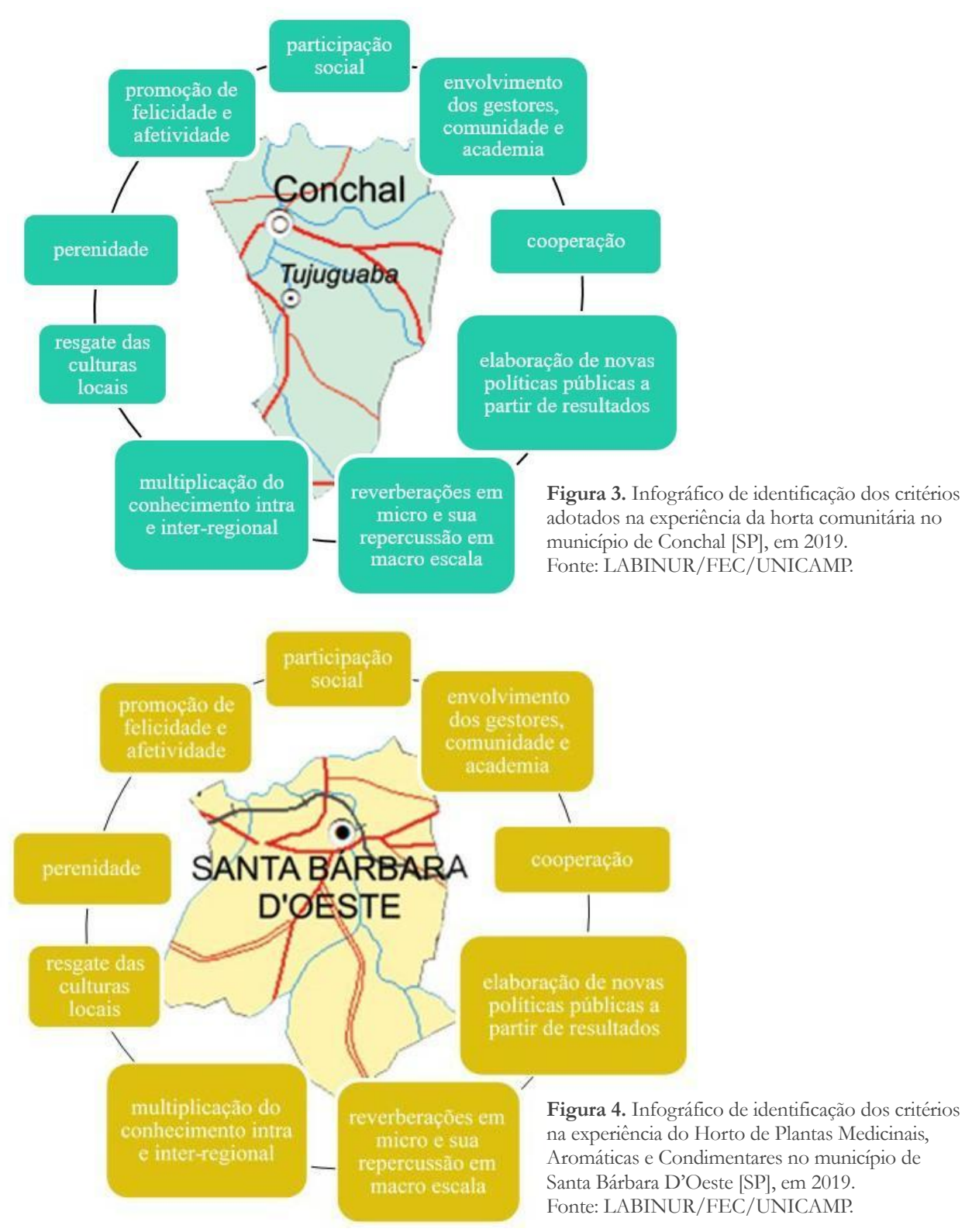

A correlação entre as práticas relatadas e o atendimento a critérios para a identificação de evidências indica que quanto maior a quantidade de critérios atendidos, maior é a participação social nos projetos desenvolvidos.

Observando as três experiências abordadas nos parágrafos acima é possível constatar particularidades importantes para o desenvolvimento de uma cidade saudável ao verificar conexões entre os princípios e valores citados na PNPS, no Estatuto da Cidade e nos documentos das ODS, baseados nas evidências que expressam efetividade, eficiência, eficácia e segurança (aspectos fundamentais da MBE).

\section{Consideracões}

A partir da teoria e prática apresentados nos estudos acima, é possível identificar as evidências das metodologias participativas no contexto do planejamento urbano e da cidade saudável. Essas evidências foram notadas considerando a integração dos campos e as conexões entre os documentos de marcos legais. Com essas e outras experiências, o LABINUR/FEC/UNICAMP tem obtido elementos a fim de construir o entendimento para a formulação de uma metodologia do Planejamento Urbano Baseado em Evidências para a Cidade Saudável que poderá ser indicada para os gestores públicos, com o intuito de relacionar a escolha ideal das iniciativas a serem postas em prática, trazer a participação social para o processo de tomada de decisões e de promover políticas públicas saudáveis a partir da experiência realizada. 
Utilizar as evidências para promoção da saúde por intermédio de ações no âmbito do planejamento urbano, exige a capacidade de aprender com as experiências, monitorar os resultados das práticas e devolver estes para as comunidades para estabelecer novos desafios.

As relações múltiplas existentes nos fenômenos urbanos demandam a ação conjunta, diversificada e positiva, para a conquista de meios que estabeleçam elementos favoráveis à criação de espaços para a "potencialização dos indivíduos" (Sperandio, 2010, p. 19) e promovam a equidade e a o sentimento de felicidade. As iniciativas apresentadas foram consideradas adequadas pelos pesquisadores, na medida que favorecem o desenvolvimento de políticas para cidade saudável. A participação social fomenta o sentimento de pertencer à cidade, resgata princípios e valores e tem importante papel na manifestação dos outros critérios apresentados neste trabalho.

É importante realizar novas pesquisas relacionados aos tópicos trazidos neste artigo, tendo em vista sua relevância estrutural na construção da cidade justa, equitativa e saudável.

O LABINUR tem obtido elementos diferenciais para subsidiar a formulação de uma nova metodologia para o planejamento urbano de uma cidade ao indicar o PUBECS (Planejamento Urbano Baseado em Evidências para a Cidade Saudável).

\section{Reierências}

Atallah, A. N. (2018). Medicina baseada em evidências. Revista Diagnóstico \& Tratamento, 23, ed. 2, pp. 43-44, 2018. São Paulo. ISSN 1413-9979.

Barten, F., Akerman, M., Becker, D., Friel, S., Hancock, T., Mwatsama, M., Rice, M., Sheuya, S., \& Stern, R. (2011). Rights, Knowledge, and Governance for Improved Health Equity in Urban Settings. Journal of urban bealth: Bulletin of the New York Academy of Medicine, 88 (5), pp. 896-905. https://doi.org/10.1007/s11524-011-9608-z

Corburn, J. (2017). Equitable and Healthy City Planning: Towards Healthy Urban Governance in the Century of the City. In: De Leeuw, E. \& Simos, J. Healthy Cities. New York: Springer.

De Leeuw, E. (2017). From Urban Projects to Healthy City Policies. In: De Leeuw, E. \& Simos, J. Healthy Cities. New York: Springer.

Domhardt, K. S. (2012). The garden city idea in the CIAM discourse on urbanism: a path to comprehensive planning. Planning Perspectives, 27 (2), pp.173-197, DOI: 10.1080/02665433.2012.646768

El Dib, R. P. (2007). Como praticar a medicina baseada em evidências. Jornal Vascular Brasileiro, 6 (1), pp.1-4. https:// dx.doi.org/10.1590/S1677-54492007000100001

Freire, M. S. M., Salles, R. P. D., \& Franco de Sá, R. M. P. (2016). Mapeando iniciativas territoriais saudáveis, suas características e evidências de efetividade. Ciência \& Saúde Coletiva, 21 (6), pp. 1757-1766. https://dx.doi.org/ 10.1590/1413-81232015216.08172016

Instituto Brasileiro de Geografia e Estatística (2019). População estimada. IBGE, Diretoria de Pesquisas, Coordenação de População e Indicadores Sociais, Estimativas da população residente com data de referência $1^{\circ}$ de julho de 2019. Cidades e Estados. Recuperado em 07 março, 2019, de https://www.ibge.gov.br/cidades-e-estados/sp/conchal.html?

Lei n. 1761, de 24 de setembro de 2010. Instituiu o programa de Políticas Públicas Saudáveis no Município de Conchal,. Recuperado em 04 de março de 2019, de https://consulta.siscam.com.br/camaraconchal/arquivo?Id=3000

Lei n. 10.257, de 10 de julho de 2001, Estatuto da Cidade e Legislação Correlata (2001). Regulamenta os arts. 182 e 183 da Constituição Federal, estabelece diretrizes gerais da política urbana e dá outras providências. Brasília: Senado Federal, Subsecretaria de Edições Técnicas. Recuperado em 04 de março de 2019, de http://www.planalto.gov.br/ ccivil_03/LEIS/LEIS_2001/L10257.htm

Lopes, A. A. (2000). Medicina Baseada em Evidências: a arte de aplicar o conhecimento científico na prática clínica. Revista da Associação Médica Brasileira, 46 (3), pp. 285-288. https://dx.doi.org/10.1590/S0104-42302000000300015

Ministério da Saúde (2002). As Cartas da Promoção da Saúde. Ministério da Saúde, Secretaria de Políticas de Saúde, Projeto Promoção da Saúde. Brasília : Ministério da Saúde.

Politica Nacional de Promoção da Saúde, Revisão da Portaria MS/GM nº 687, de 30 de março de 2006 (2014). Redefine a Política Nacional de Promoção da Saúde (PNPS). Brasília. Recuperado em 04 de março de 2019 de http:// bvsms.saude.gov.br/bvs/publicacoes/pnps_revisao_portaria_687.pdf

Prefeitura Municipal de Conchal (2018). Ata Reunião Horta Comunitária de 25 de janeiro de 2018. Conchal: Departamento de Saúde da Prefeitura Municipal de Conchal.

Rheingantz, P. A., Azevedo, G. A., Brasileiro, A., Alcantara, D. \& Queiroz, M. (2009). Observando a qualidade do lugar: procedimentos para a avaliação pós-ocupação. Rio de Janeiro: Universidade Federal do Rio de Janeiro. 
Rogers, R. G., \& Gumuchdjiam, P. (2001). Cidades para um pequeno planeta. Barcelona: Gustavo Gili Brasil.

Snowden, D., \& Boone, M. (2007). A Leader's Framework for Decision Making. Harvard business review. 85. 68-76, 149. ISSN: 0017-8012.

Sperandio, A. M. G. (2010). A promoção da saúde construída em rede (pp. 19-21). In : Sperandio, A. M. G., Machin, D. G., \& Fortunato, M. A. B. (Orgs.), Políticas Integradas em Rede e a Construção de Espaços Saudáveis: boas práticas para a Iniciativa do Rostos, Vozes e Lugares. Brasília: Organização Pan Americana da Saúde.

Sperandio, A. M. G., Francisco Filho, L. L., Favero, E., Ribeiro, M. M., \& Manfrinato, T. (2015). Ocupação de vazio urbano como promotor do planejamento para cidade saudável. PARC Pesquisa em Arquitetura e Construção, 6 (3), pp. 205-215. https://doi.org/10.20396/parc.v6i3.8635018

Sperandio, A. M. G., Malek-Zadeh, M. U., Arêas, J. L. de S., \& Francisco Filho, L. L. (2019). Mapa dos desejos locais como uma ferramenta para promoção do diálogo e do desenvolvimento de cidades saudáveis. PARC Pesquisa em Arquitetura e Construção, 10, e019002. https://doi.org/10.20396/parc.v10i0.8652312

Sperandio, A. M. G., Moreira, R. N., \& Bernardino, F. (2018). Conexões entre o planejamento urbano e a cidade saudável. Labor \& Engenho, 12(4), pp. 482-494. https://doi.org/10.20396/labore.v12i4.8654239

Sperandio, A. M. G., Rosa, A. A. C., \& Carvalho, B. G. (2017). Reverberação Política e Educativa para Cidade Saudável. Cadernos de Pós-Graduação em Arquitetura e Urbanismo, 17 (2), p. 26. DOI 10.5935/cadernosarquitetura.v17n2p26-51.

Streck, D. R. (2016). Metodologias participativas de pesquisa e educação popular: reflexões sobre critérios de qualidade. Interface — Comunicacão, Saúde, Educação, 20(58), 537-547. Epub March 01, 2016.https://dx.doi.org/ $10.1590 / 1807-57622015.0443$

Weiss, M. C. (2017). Os desafios a gestão das cidades: uma chamada para a ação em tempos de emergência das cidades inteligentes no Brasil. Direito da Cidade, 9 (2), pp. 788-824. [s. 1.]. DOI: 10.12957/rdc.2017.27493. 